\title{
Determination of antagonistic effect of bio- agents against cereal damping-off caused by Fusarium graminearum in wheat
}

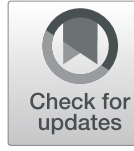

Yesim Er

\begin{abstract}
The antagonistic effect of the isolates of Gliocladium roseum, Saccharomyces cerevisiae, Sordaria fimicola, and their mixtures, at different concentrations against the cereal damping-off pathogen, Fusarium graminearum, was examined in vitro and in vivo (foliar, seed, soil, seed + soil) treatments on the susceptible wheat cultivars "Gun 91 and Sultan 95." The 3 isolates inhibited growth of $F$. graminearum at a concentration of $1 \times 10^{9} \mathrm{spores} / \mathrm{ml}$ with inhibition rates of 84,88 , and $91 \%$, respectively under in vitro conditions. For in vivo assays, the mixture of S. cerevisiae + S. fimicola exhibited a considerable antagonistic activity even at a concentration of $1 \times 10^{5} \mathrm{spores} / \mathrm{ml}$. Particularly, at the seed + soil treatment of the mixture, the pathogen was almost completely suppressed with an inhibition rate above $96 \%$ at concentrations of $1 \times 10^{8}$ and $1 \times 10^{9}$ spores $/ \mathrm{ml}$ for both wheat cultivars, and the percentage of emerged seedlings reached nearly $100 \%$. The results verified that the mixture of S. cerevisiae + S. fimicola had a high potential, as a promising biocontrol agents and an eco-friendly alternative, to be used against the cereal damping-off caused by F. graminearum, to reduce the use of systemic fungicides.
\end{abstract}

Keywords: Antagonistic effect, Bio-agent, Wheat, Cereal damping-off pathogen, Fusarium graminearum

\section{Background}

The diseases caused by plant pathogenic bacteria and fungi cause significant reductions in crop yield and huge product losses worldwide (Avelino et al. 2015). Wheat is one of the most important cereal crops as staple food for more than 2 billion people. Many phytopathogens, including Fusarium graminearum, reduce the yield quantity and quality in cereal crops and cause severe economic losses throughout the world (Kelly and Ward 2018). This pathogen infects wheat seeds and causes damping-off (Rasiukeviciute and Kelpsiene 2018), which decreases seed germination and seedling emergence in regions with mild and semitropical climates. F. graminearum produces sexual spores (ascospores) and asexual spores (macroconidia). The primary infection by ascospores appears in the spring (Leplat et al. 2013).

Correspondence: y_err@hotmail.com

Department of Plant Pathology, Istanbul Directorate of Agricultural Quarantine, Istanbul, Turkey
Also, the pathogen can produce a variety of mycotoxins, threatening human health seriously (Leplat et al. 2013 and Omotayo et al. 2019).

The efforts to develop management methods against the disease are not satisfactory and promising. Indeed, non-chemical methods, such as crop residue removal, crop rotation, pathogen free-seed, and cultivation are not the solution for cereal-intensive areas (Peltonen-Sainio et al. 2009 and Vinale et al. 2009). Furthermore, the efficacy of these practices does not contribute to control the disease due to environmental conditions stimulating pathogen growth and disease incidence (Perez et al. 2008 and Landschoot et al. 2013).

Recently, chemical control against plant diseases is proposed as the most effective method. The unconscious and overuse of pesticides causes environmental pollution and degradation of the ecosystem. Besides, the resistance of target organisms as a result of long-term use of pesticides is another disadvantage. Moreover, systemic 
fungicides can be easily absorbed by soil, causing pollution of foods and environment (Schaafsma et al. 2005 and Satapute et al. 2019). Biological control of plant pathogens such as by $F$. graminearum may provide feasible alternatives instead of synthetic fungicides (Jogaiah et al. 2018 and Joshi et al. 2019).

A few documented studies about the possible antagonistic activity of Gliocladium roseum, Saccharomyces cerevisiae, and Sordaria fimicola have been reported against Fusarium spp. For instance, G. roseum was revealed as a soil-borne endophyte against Fusarium spp. (Zhang et al. 2008 and Hue et al. 2009), stimulating plant growth against $F$. culmorum in winter wheat (Knudsen et al. 1995) and reducing the colonization of Fusarium species in wheat and maize by suppressing sporulation production (Luongo et al. 2005 and Woo et al. 2006). S. cerevisiae was reported as a good producer of antifungal antibiotics against growth of Fusarium spp. (Suzzi et al. 1995), promoting seed germination and plant growth due to indole-3-acetic acid (IAA) producing ability on tomato and eggplant seedlings (Attyia and Youssry 2001). The antagonistic activity of S. fimicola was only reported in maize against $F$. graminearum under greenhouse conditions (Abdallah et al. 2018).

The present study aimed to evaluate the antagonistic activity of 3 isolates; Gliocladium roseum, Saccharomyces cerevisiae, and Sordaria fimicola and to determine the most effective antagonistic agents against F. graminearum in susceptible wheat cultivars.

\section{Materials and methods Materials}

The pathogen was isolated from wheat seed coats of the infected samples. Potato dextrose agar (PDA) medium was used for the isolation and cultivation of the pathogen. Rose Bengal agar (RBA) and PDA were used for the isolation and cultivation of the antagonists obtained from soil samples. The soil samples were taken at $15-\mathrm{cm}$ depth from rhizosphere to obtain a high diversity of antagonists and from 15 various areas in the province of Tekirdag (Turkey) throughout September 2018. The seeds of the susceptible wheat cultivars "Gun 91 and Sultan 95" were purchased and used to determine the antagonistic activity of the bio-agent treatments against $F$. graminearum. The study was conducted in vitro and in vivo assays (foliar, seed, soil and seed + soil treatments) at 5 concentrations of $1 \times 10^{5}$ to $1 \times 10^{9}$ spores $/ \mathrm{ml}$. In vivo assays were carried out using 30 samples of the wheat seeds per pot $(22 \times 15$-cm diameter $)$ containing $100 \mathrm{~g}$ sterile peat.

\section{Isolation and identification of the causal pathogen and the bio-agents}

The wheat seed samples were disinfected by $1 \%$ sodium hypochlorite solution (SHS) for $5 \mathrm{~min}$ and rinsed 3 times with sterile distilled water (SDW). After drying process on sterile filter paper, the seeds were placed onto PDA plates and incubated at $28^{\circ} \mathrm{C}$ for 7 days. After incubation period, the fungal isolates were purified by a single spore technique and kept at $-10{ }^{\circ} \mathrm{C}$ throughout the study. The purified isolates were identified according to cultural, morphological (Ellis 1971 1976; Booth 1971 1977; Karaca 1974; Domsch et al. 1980; Nelson et al. 1981; Burgess et al. 1994), microscopic (Nelson et al. 1981; Toussoun and Nelson 1995), and pathological (Nelson et al. 1981; Singh et al. 1991; Toussoun and Nelson 1995) properties. The pathogenicity of 20 isolates of F. graminearum was pre-assessed on the basis of the number of dead seedlings of both wheat cultivars after 15 days under plant growth room conditions (at $25^{\circ} \mathrm{C}, 16 \mathrm{~h}$ of photoperiod and $100 \% \mathrm{RH}$ ) to detect the most pathogenic fungal isolate for the next experiments. The experiment was carried out according to the same procedure as in vivo assays.

The soil samples were sieved and dried for a week at $18^{\circ} \mathrm{C}$ and then kept at $10^{\circ} \mathrm{C}$. The antagonistic microbial flora was incubated for 7-9 days and isolated with serial dilution technique (Rai and Kovics 2010) on RBA (dextrose, $10 \mathrm{~g}$; soy peptone, $5 \mathrm{~g}$; $\mathrm{KH}_{3} \mathrm{PO}_{4}, 1 \mathrm{~g} ; \mathrm{MgSO}_{4}, 0.5 \mathrm{~g}$; streptomycin sulfate salt, $1.0 \mathrm{~g}$; Rose Bengal, $0.05 \mathrm{~g}$; agar, $15 \mathrm{~g}$; chloramphenicol, $0.1 \mathrm{~g}$; distilled water, $1000 \mathrm{ml}$ ) for

Table 1 Effect of the bio-agents on in vitro mycelial growth of F. graminearum

\begin{tabular}{|c|c|c|c|c|c|c|}
\hline \multirow[b]{2}{*}{$\begin{array}{l}\text { Concentrations (spores/ } \\
\mathrm{ml} \text { ) }\end{array}$} & \multicolumn{2}{|l|}{ G. roseum } & \multicolumn{2}{|l|}{ S. cerevisiae } & \multicolumn{2}{|l|}{ S. fimicola } \\
\hline & $\begin{array}{l}\text { Mycelial growth } \\
(\mathrm{cm})\end{array}$ & $\begin{array}{l}\text { Inhibition rate } \\
(\%)\end{array}$ & $\begin{array}{l}\text { Mycelial growth } \\
(\mathrm{cm})\end{array}$ & $\begin{array}{l}\text { Inhibition rate } \\
(\%)\end{array}$ & $\begin{array}{l}\text { Mycelial growth } \\
(\mathrm{cm})\end{array}$ & $\begin{array}{l}\text { Inhibition rate } \\
(\%)\end{array}$ \\
\hline $1 \times 10^{5}$ & $4.7 \pm 0.56 c^{*}$ & 53.0 & $4.0 \pm 0.87 d^{*}$ & 60.0 & $3.7 \pm 0.68 d e^{*}$ & 67.0 \\
\hline $1 \times 10^{6}$ & $3.9 \pm 0.45 \mathrm{~cd}$ & 61.0 & $3.3 \pm 0.74 \mathrm{de}$ & 67.0 & $3.0 \pm 0.21 \mathrm{e}$ & 70.0 \\
\hline $1 \times 10^{7}$ & $3.2 \pm 0.85 d$ & 68.0 & $2.6 \pm 0.25 e$ & 74.0 & $2.1 \pm 0.74 \mathrm{ef}$ & 79.0 \\
\hline $1 \times 10^{8}$ & $2.5 \pm 0.21 \mathrm{de}$ & 75.0 & $2.0 \pm 0.22 \mathrm{ef}$ & 80.0 & $1.5 \pm 0.26 \mathrm{efg}$ & 85.0 \\
\hline $1 \times 10^{9}$ & $1.6 \pm 0.18 \mathrm{e}$ & 84.0 & $1.2 \pm 0.10 f$ & 88.0 & $0.9 \pm 0.12 g$ & 91.0 \\
\hline Control & $10.0 \pm 0.91 a$ & - & $10.0 \pm 0.83 a$ & - & $10.0 \pm 0.74 a$ & - \\
\hline
\end{tabular}

*Values expressed are mean ( \pm standard error) of five replicates

Values given separately for in vitro treatments within each column followed by different letters are significantly different at $p<0.05$ 
growth of antagonistic yeasts, and on PDA (agar, $15.0 \mathrm{~g}$; dextrose, $20.0 \mathrm{~g}$; potato extract, $4.0 \mathrm{~g}$; streptomycin sulfate salt, $1.0 \mathrm{~g}$; distilled water, $1000 \mathrm{ml}$ ) for growth of fungal antagonists. Consequently, the single spore or hyphal tip was taken from the developing fungal colonies and transferred onto PDA medium containing streptomycin sulfate salt $(1.0 \mathrm{~g} / \mathrm{l})$ and incubated at room temperature $\left(25 \pm 2{ }^{\circ} \mathrm{C}\right)$ for a week. The selected 30 antagonistic isolates were identified according to mycological keys (Lundquist 1972; Vaughan Martini and Martini 1993 and Gams and Bissett 1998). In this study, a preliminary experiment was conducted to assess the identified isolates exhibiting the highest antagonistic activity against $F$. graminearum on PDA medium. The experiment was carried out according to the same procedure as in vitro assays. As a result of the assessment, the most antagonistic isolates were selected and used for the next experiments. In order to prepare the spore suspensions, the pathogen cultures were passed through 2 layers of cheesecloth, diluted with sterile distilled water to a concentration of $1 \times 10^{5}$ conidia $/ \mathrm{ml}$ and centrifuged at $4.000 \mathrm{rpm}$ for $5 \mathrm{~min}$ at $22^{\circ} \mathrm{C}$. The cultures of the bio-agents were blended in an electric blender for $2 \mathrm{~min}$ and prepared as a liquid suspension with sterile distilled water. All bio-agents were adjusted at 5 different concentrations from $1 \times 10^{5}$ to $1 \times 10^{9}$ spores $/ \mathrm{ml}$ and centrifuged at $5500 \mathrm{rpm}$ for $5 \mathrm{~min}$ for S. cerevisiae (Janson and Elshadei 2012) and at $2500 \mathrm{rpm}$ for $10 \mathrm{~min}$ for G. roseum and S. fimicola. The obtained supernatants were filtered through grade no. 1 Whatman filter paper and used for in vitro and in vivo assays.

\section{Determination of the antagonistic activity of the bio- agents (in vitro)}

One disc (0.5-cm diameter) of 7-day-old culture of the pathogen isolate was placed to the center of the plate. The adjusted spore suspensions of G. roseum, S. cerevisiae, and $S$. fimicola were streaked parallel on either side of the fungal disc at a distance of $2 \mathrm{~cm}$ (Jalaluldeen et al. 2014). In addition, a disc $(0.5 \mathrm{~cm})$ of G. roseum-S. cerevisiae, G. roseum-S. fimicola and, S. cerevisiae-S. fimicola was placed at a distance of $2 \mathrm{~cm}$ from PDA plate edge to evaluate the antagonistic interaction between each other. The plates were incubated at $25^{\circ} \mathrm{C}$ for 7-9 days. After the incubation period, the diameter of each colony was measured to evaluate the antagonistic activity as a result of the average of 5 independent replicates. The antagonist-free PDA medium, containing only SDW and a culture disk of the pathogen, was used as a control. The effect of the bio-agent treatments on mycelial growth of the pathogenic fungus was determined after 7 days and calculated using the following formula (Topps and Wain 1957).

$$
I \%=[(C-T) / C] \times 100
$$

where I \% = inhibition rate, $\mathrm{C}=$ average diameter of mycelial growth of pathogenic fungus in control

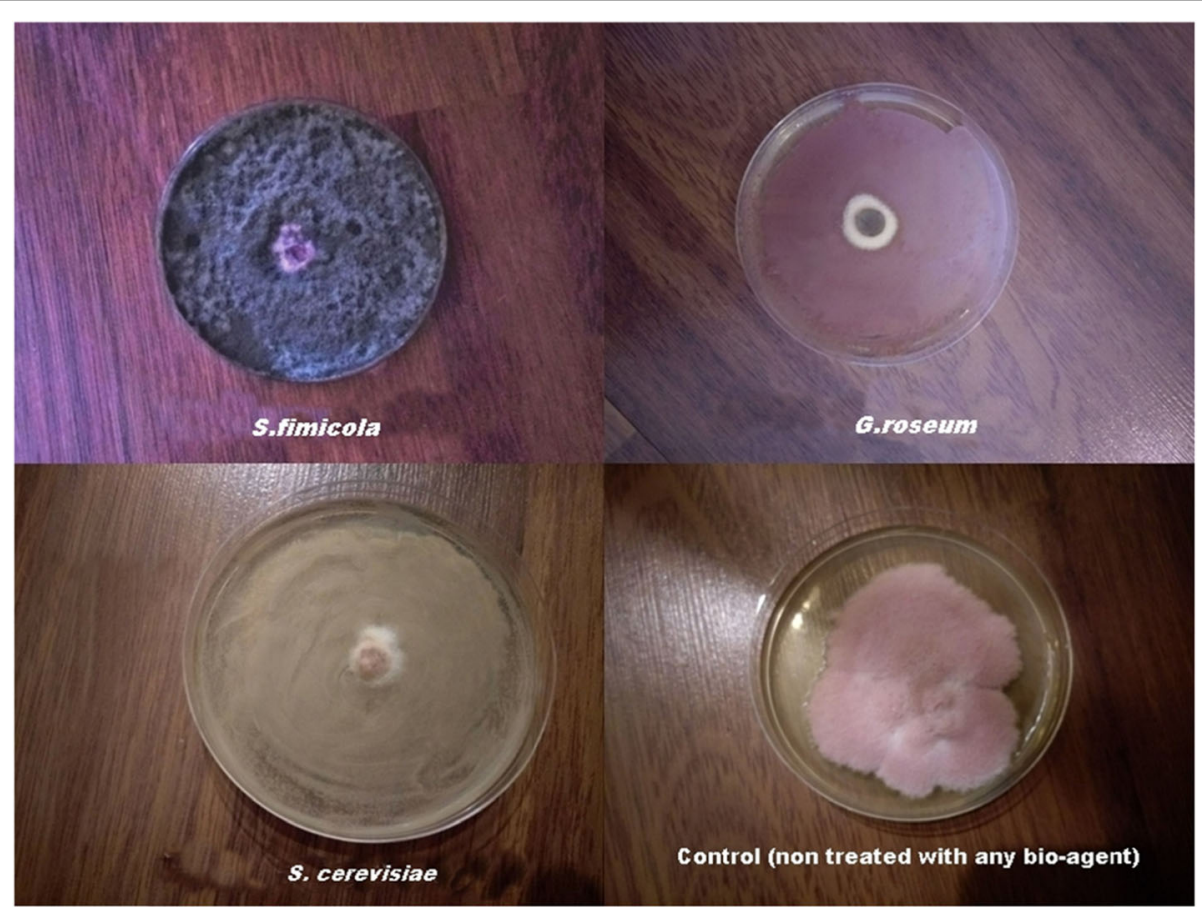

Fig. 1 The mycelial growth of $F$. graminearum after in vitro treatments with the bio-agents at $1 \times 10^{9}$ spores $/ \mathrm{ml}$ and the control treatment 
treatment, and $\mathrm{T}=$ average diameter of mycelial growth of pathogenic fungus after bio-agent treatments.

\section{Determination of in vivo antagonistic activity of the bio- agents on leaf infection}

The wheat seed samples were disinfected by $1 \%$ SHS for 5 min and rinsed 3 times with SDW before bio-agent treatments. The seeds of wheat cultivars "Gun 91and Sultan 95" were planted in experimental pots $(22 \times 15$ $\mathrm{cm}$ diameter) containing a sterile peat and were grown in the plant growth room conditions. When plants reached the two-leaf stage in 2 weeks, G. roseum, S. cerevisiae, S. fimicola, and a mixture of the bio-agents (G. roseum + S. cerevisiae, G. roseum + S. fimicola, S. cerevisiae + S. fimicola) were sprayed as a foliar treatment separately at concentrations of $1 \times 10^{5}$ to $1 \times 10^{9}$ spores $/ \mathrm{ml}$ to upper and lower surfaces of wheat leaves with a doseadjusted spray at the rate of $0.2 \mathrm{ml}$ per leaf. After the inocula were allowed to dry on the leaves (approximately $1-2 \mathrm{~h}$ ), the pathogen inoculum at a concentration of $1 \times$ $10^{5}$ conidia/ml was applied to wheat leaves in the same way. Following the inoculation process, the plants were

Table 2 Effect of different concentrations of the foliar treatments on lesion length caused by F. graminearum in wheat cultivars

\begin{tabular}{|c|c|c|c|c|c|}
\hline \multirow[t]{3}{*}{ Foliar treatments } & \multirow{3}{*}{$\begin{array}{l}\text { Concentrations } \\
\text { (spores/ml) }\end{array}$} & \multicolumn{4}{|l|}{ Wheat cultivars } \\
\hline & & \multicolumn{2}{|l|}{ Gun 91} & \multicolumn{2}{|l|}{ Sultan 95} \\
\hline & & Lesion length (mm) & Inhibition rate (\%) & Lesion length (mm) & Inhibition rate (\%) \\
\hline \multirow[t]{5}{*}{ G. roseum } & $1 \times 10^{5}$ & $9.7 \pm 1.56 c^{*}$ & 34.4 & $8.4 \pm 0.90 \mathrm{~cd}^{*}$ & 36.3 \\
\hline & $1 \times 10^{6}$ & $7.7 \pm 0.90 d$ & 47.9 & $6.7 \pm 0.81 d$ & 49.2 \\
\hline & $1 \times 10^{7}$ & $6.7 \pm 1.25 \mathrm{~d}-\mathrm{h}$ & 54.7 & $5.8 \pm 0.31 e$ & 56.0 \\
\hline & $1 \times 10^{8}$ & $5.6 \pm 0.67 \mathrm{e}-\mathrm{g}$ & 62.1 & $4.3 \pm 0.81 f-h$ & 67.4 \\
\hline & $1 \times 10^{9}$ & $3.5 \pm 0.12 \mathrm{~g}$ & 76.3 & $2.9 \pm 0.21 \mathrm{~h}$ & 78.0 \\
\hline \multirow[t]{5}{*}{ S. cerevisiae } & $1 \times 10^{5}$ & $8.9 \pm 0.57 \mathrm{~cd}$ & 39.8 & $7.8 \pm 0.55 c-e$ & 40.9 \\
\hline & $1 \times 10^{6}$ & $7.0 \pm 0.42 \mathrm{~d}-\mathrm{g}$ & 52.7 & $6.1 \pm 0.90 \mathrm{def}$ & 53.7 \\
\hline & $1 \times 10^{7}$ & $5.7 \pm 0.71 \mathrm{e}-\mathrm{g}$ & 61.4 & $5.0 \pm 0.45 \mathrm{ef}$ & 62.1 \\
\hline & $1 \times 10^{8}$ & $4.5 \pm 0.21 \mathrm{fg}$ & 69.5 & $3.9 \pm 0.05 \mathrm{~g}$ & 70.4 \\
\hline & $1 \times 10^{9}$ & $2.7 \pm 0.73 \mathrm{hi}$ & 81.7 & $2.1 \pm 0.01 \mathrm{~h}-\mathrm{j}$ & 84.0 \\
\hline \multirow[t]{5}{*}{ S. fimicola } & $1 \times 10^{5}$ & $8.5 \pm 0.43 c-e$ & 42.5 & $7.3 \pm 0.87 c-f$ & 44.6 \\
\hline & $1 \times 10^{6}$ & $6.8 \pm 0.97 d-h$ & 54.0 & $5.7 \pm 0.56 e$ & 56.8 \\
\hline & $1 \times 10^{7}$ & $5.5 \pm 0.35 \mathrm{e}-\mathrm{g}$ & 62.8 & $4.6 \pm 0.41 \mathrm{fg}$ & 65.1 \\
\hline & $1 \times 10^{8}$ & $4.0 \pm 1.08 f-i$ & 72.9 & $3.2 \pm 0.16 \mathrm{~g}-\mathrm{i}$ & 75.7 \\
\hline & $1 \times 10^{9}$ & $2.4 \pm 0.65 h-j$ & 83.7 & $1.8 \pm 0.06 i$ & 86.3 \\
\hline \multirow[t]{5}{*}{ G. roseum + S. cerevisiae } & $1 \times 10^{5}$ & $9.1 \pm 0.78 \mathrm{~cd}$ & 38.5 & $8.0 \pm 0.59 c-e$ & 39.3 \\
\hline & $1 \times 10^{6}$ & $7.4 \pm 0.43 \mathrm{~d}-\mathrm{f}$ & 50.0 & $6.6 \pm 0.32 d$ & 50.0 \\
\hline & $1 \times 10^{7}$ & $6.0 \pm 0.79 \mathrm{ef}$ & 59.4 & $5.1 \pm 0.68 \mathrm{ef}$ & 61.3 \\
\hline & $1 \times 10^{8}$ & $4.9 \pm 0.41 f$ & 66.8 & $3.5 \pm 0.52 \mathrm{gh}$ & 73.4 \\
\hline & $1 \times 10^{9}$ & $3.1 \pm 0.70 \mathrm{~g}-\mathrm{i}$ & 79.0 & $2.4 \pm 0.21 \mathrm{hi}$ & 81.8 \\
\hline \multirow[t]{5}{*}{ G. roseum + S. fimicola } & $1 \times 10^{5}$ & $9.1 \pm 0.83 \mathrm{~cd}$ & 38.5 & $7.9 \pm 0.45 c-e$ & 40.1 \\
\hline & $1 \times 10^{6}$ & $7.2 \pm 0.50 \mathrm{~d}-\mathrm{g}$ & 51.3 & $6.2 \pm 0.94$ def & 53.0 \\
\hline & $1 \times 10^{7}$ & $5.9 \pm 0.35 \mathrm{ef}$ & 60.1 & $5.0 \pm 0.41 \mathrm{ef}$ & 62.1 \\
\hline & $1 \times 10^{8}$ & $4.7 \pm 0.79 f g$ & 68.2 & $3.6 \pm 0.91 \mathrm{gh}$ & 72.7 \\
\hline & $1 \times 10^{9}$ & $2.9 \pm 0.72 h$ & 80.4 & $2.3 \pm 0.15 \mathrm{hi}$ & 82.5 \\
\hline \multirow[t]{5}{*}{ S. cerevisiae + S. fimicola } & $1 \times 10^{5}$ & $6.0 \pm 0.70 \mathrm{ef}$ & 59.4 & $5.1 \pm 0.95 \mathrm{ef}$ & 61.3 \\
\hline & $1 \times 10^{6}$ & $4.9 \pm 0.68 f$ & 66.8 & $4.0 \pm 0.41 f-i$ & 69.6 \\
\hline & $1 \times 10^{7}$ & $3.2 \pm 0.92 \mathrm{gh}$ & 78.3 & $2.3 \pm 0.91 \mathrm{hi}$ & 82.5 \\
\hline & $1 \times 10^{8}$ & $1.9 \pm 0.06 \mathrm{ij}$ & 87.1 & $1.2 \pm 0.05 i j$ & 90.9 \\
\hline & $1 \times 10^{9}$ & $1.1 \pm 0.04 j$ & 92.5 & $0.6 \pm 0.01 j$ & 94.4 \\
\hline Control & - & $14.8 \pm 1.34 a$ & - & $13.2 \pm 1.40 \mathrm{a}$ & - \\
\hline
\end{tabular}

*Values expressed are mean ( \pm standard error) of five replicates

Values given separately for the foliar treatments within each column followed by different letters are significantly different at $p<0.05$ 
kept in a plant growth room to provide optimum conditions for disease development for $72 \mathrm{~h}$. Afterwards, the seedlings were kept at room temperature for $24 \mathrm{~h}$ (Kawasaki 2008). Consequently, the leaves were detached separately from the plants, and the disease incidence was determined according to visually necrotic lesion and/or sporulation area of the disease. The lesion area was measured as the average of 5 independent replicates and compared with the control pots sprayed with a spore suspension of the fungal pathogen only. The effect of the bio-agent treatments on leaf infection was calculated using the formula of Topps and Wain (1957).

$$
I \%=[(C-T) / C] \times 100
$$

where I \% = inhibition rate, $\mathrm{C}=$ average lesion length in control treatment, and $\mathrm{T}=$ average lesion length after bio-agent treatments.

Table 3 Effect of different concentrations of the seed treatments on damping-off caused by F. graminearum in wheat cultivars

\begin{tabular}{|c|c|c|c|c|c|}
\hline \multirow[t]{3}{*}{ Seed treatments } & \multirow{3}{*}{$\begin{array}{l}\text { Concentrations } \\
\text { (spores/ml) }\end{array}$} & \multicolumn{4}{|l|}{ Wheat cultivars } \\
\hline & & \multicolumn{2}{|l|}{ Gun 91} & \multicolumn{2}{|l|}{ Sultan 95} \\
\hline & & Incidence of damping-off (\%) & Inhibition rate (\%) & Incidence of damping-off (\%) & Inhibition rate (\%) \\
\hline \multirow[t]{5}{*}{ G. roseum } & $1 \times 10^{5}$ & $45.7 \pm 0.24 c-e^{*}$ & 54.3 & $40.0 \pm 0.25 \mathrm{~cd}^{*}$ & 60.0 \\
\hline & $1 \times 10^{6}$ & $40.3 \pm 0.83 d$ & 59.7 & $33.0 \pm 0.44 \mathrm{de}$ & 67.0 \\
\hline & $1 \times 10^{7}$ & $33.7 \pm 0.44 \mathrm{e}-\mathrm{g}$ & 66.3 & $28.3 \pm 0.84 \mathrm{e}-\mathrm{h}$ & 71.7 \\
\hline & $1 \times 10^{8}$ & $26.1 \pm 0.21 \mathrm{f}-\mathrm{h}$ & 73.9 & $22.4 \pm 0.31 f-i$ & 77.6 \\
\hline & $1 \times 10^{9}$ & $18.5 \pm 0.45 g-i$ & 81.5 & $14.2 \pm 0.79 \mathrm{hi}$ & 85.8 \\
\hline \multirow[t]{5}{*}{ S. cerevisiae } & $1 \times 10^{5}$ & $49.0 \pm 0.92 b-f$ & 51.0 & $41.2 \pm 0.45 c$ & 58.8 \\
\hline & $1 \times 10^{6}$ & $32.7 \pm 0.12 \mathrm{e}-\mathrm{h}$ & 67.3 & $31.8 \pm 0.90 \mathrm{~d}-\mathrm{g}$ & 68.2 \\
\hline & $1 \times 10^{7}$ & $27.2 \pm 0.44 \mathrm{fg}$ & 72.8 & $23.6 \pm 0.87 f-h$ & 76.4 \\
\hline & $1 \times 10^{8}$ & $20.7 \pm 0.84 \mathrm{~g}$ & 79.3 & $16.5 \pm 0.61 \mathrm{~g}-j$ & 83.5 \\
\hline & $1 \times 10^{9}$ & $14.1 \pm 0.10 h-j$ & 85.9 & $9.5 \pm 0.28 i-k$ & 90.5 \\
\hline \multirow[t]{5}{*}{ S. fimicola } & $1 \times 10^{5}$ & $43.5 \pm 0.89 c-g$ & 56.5 & 35.304.91c-h & 64.7 \\
\hline & $1 \times 10^{6}$ & $37.0 \pm 0.44 d-h$ & 63.0 & $29.5 \pm 0.21 \mathrm{e}-\mathrm{g}$ & 70.5 \\
\hline & $1 \times 10^{7}$ & $31.6 \pm 0.23 \mathrm{e}-\mathrm{i}$ & 68.4 & $23.6 \pm 0.13 f-h$ & 76.4 \\
\hline & $1 \times 10^{8}$ & $21.8 \pm 0.90 f-1$ & 78.2 & $16.5 \pm 0.91 \mathrm{~g}-j$ & 83.5 \\
\hline & $1 \times 10^{9}$ & $15.3 \pm 0.36 h i$ & 84.7 & $11.8 \pm 0.21 \mathrm{i}$ & 88.2 \\
\hline \multirow[t]{5}{*}{ G. roseum + S. cerevisiae } & $1 \times 10^{5}$ & $46.8 \pm 0.71 \mathrm{~cd}$ & 53.2 & $38.9 \pm 0.60 c-e$ & 61.1 \\
\hline & $1 \times 10^{6}$ & $35.9 \pm 0.67 d-k$ & 64.1 & $33.0 \pm 0.13 \mathrm{de}$ & 67.0 \\
\hline & $1 \times 10^{7}$ & $30.0 \pm 0.61 e-j$ & 70.6 & $25.9 \pm 0.61 f$ & 74.1 \\
\hline & $1 \times 10^{8}$ & $20.7 \pm 0.41 \mathrm{~g}$ & 79.3 & $18.9 \pm 0.69 \mathrm{gh}$ & 81.1 \\
\hline & $1 \times 10^{9}$ & $16.4 \pm 0.21 \mathrm{~g}-\mathrm{k}$ & 83.6 & $10.6 \pm 0.11 \mathrm{ij}$ & 89.4 \\
\hline \multirow[t]{5}{*}{ G. roseum + S. fimicola } & $1 \times 10^{5}$ & $44.6 \pm 0.78 c-f$ & 55.4 & $37.7 \pm 0.91 \mathrm{c}-\mathrm{f}$ & 62.3 \\
\hline & $1 \times 10^{6}$ & $38.1 \pm 0.81 d-g$ & 61.9 & $30.6 \pm 0.50 \mathrm{ef}$ & 69.4 \\
\hline & $1 \times 10^{7}$ & $32.7 \pm 0.11 \mathrm{e}-\mathrm{h}$ & 67.3 & $24.8 \pm 0.31 \mathrm{fg}$ & 75.2 \\
\hline & $1 \times 10^{8}$ & $24.0 \pm 0.21 f-i$ & 76.0 & $18.9 \pm 0.26 \mathrm{gh}$ & 81.1 \\
\hline & $1 \times 10^{9}$ & $17.4 \pm 0.10 \mathrm{~g}-\mathrm{j}$ & 82.6 & $13.0 \pm 0.20 h-j$ & 87.0 \\
\hline \multirow[t]{5}{*}{ S. cerevisiae + S. fimicola } & $1 \times 10^{5}$ & $29.4 \pm 0.47 e-j$ & 70.6 & $23.6 \pm 0.82 f-h$ & 76.4 \\
\hline & $1 \times 10^{6}$ & $22.9 \pm 0.89 \mathrm{fk}$ & 77.1 & $15.3 \pm 0.41 \mathrm{~g}-\mathrm{k}$ & 84.7 \\
\hline & $1 \times 10^{7}$ & $13.1 \pm 0.67 \mathrm{~h}-\mathrm{k}$ & 86.9 & $8.3 \pm 0.17 \mathrm{i}-1$ & 91.7 \\
\hline & $1 \times 10^{8}$ & $7.7 \pm 0.31 j$ & 92.3 & $3.7 \pm 0.11 \mathrm{j}-1$ & 96.3 \\
\hline & $1 \times 10^{9}$ & $2.2 \pm 0.11 \mathrm{~lm}$ & 97.8 & $1.2 \pm 0.03 \mathrm{kl}$ & 98.8 \\
\hline Control & - & $61.3 \pm 1.11 \mathrm{a}$ & - & $56.6 \pm 1.03 a$ & - \\
\hline
\end{tabular}


Determination of in vivo antagonistic activity of the bioagents on damping-off

To evaluate the antagonistic activity of the bio-agents against $F$. graminearum, different concentrations of each of G. roseum, S. cerevisiae, S. fimicola, and their mixtures (G. roseum + S. cerevisiae, G. roseum + S. fimicola, S. cerevisiae + S. fimicola) were examined as the seed, soil, and seed + soil treatments.

To determine the antagonistic activity of the seed treatments against damping-off in wheat cultivars, the pathogen inoculum at the rate of $5 \% \mathrm{w} / \mathrm{v}$ (Hussein
1973) was transferred to pots containing an autoclaved sterile peat, mixed thoroughly, and kept by moisturizing for 7 days. After the incubation period, the bio-agent suspensions were applied at different concentrations to the disinfected wheat seeds by spraying with a dose-adjusted spray to cover the seed surface homogenously at the rate of $20 \mathrm{ml} / \mathrm{kg}$ of seeds before sowing in pots.

To determine the antagonistic activity of the soil treatments, the pathogen inoculum at the rate of $5 \%$ $\mathrm{w} / \mathrm{v}$ (Hussein 1973) and the spore suspensions of

Table 4 Effect of different concentrations of the soil treatments on damping-off caused by F. graminearum in wheat cultivars

\begin{tabular}{|c|c|c|c|c|c|}
\hline \multirow[t]{3}{*}{ Soil treatments } & \multirow{3}{*}{$\begin{array}{l}\text { Concentrations } \\
\text { (spores } / \mathrm{ml} \text { ) }\end{array}$} & \multicolumn{4}{|l|}{ Wheat cultivars } \\
\hline & & \multicolumn{2}{|l|}{ Gun 91} & \multicolumn{2}{|l|}{ Sultan 95} \\
\hline & & Incidence of damping-off (\%) & Inhibition rate (\%) & Incidence of damping-off (\%) & Inhibition rate (\%) \\
\hline \multirow[t]{5}{*}{ G. roseum } & $1 \times 10^{5}$ & $51.1 \pm 0.25 b-d^{*}$ & 48.9 & $45.9 \pm 0.41 b-d^{*}$ & 54.1 \\
\hline & $1 \times 10^{6}$ & $43.5 \pm 0.31 c-g$ & 56.5 & $38.9 \pm 0.87 \mathrm{c}-\mathrm{e}$ & 61.1 \\
\hline & $1 \times 10^{7}$ & $38.1 \pm 0.96 \mathrm{~d}-\mathrm{g}$ & 61.9 & $30.6 \pm 0.22 \mathrm{ef}$ & 69.4 \\
\hline & $1 \times 10^{8}$ & $31.6 \pm 0.33 e-i$ & 68.4 & $25.9 \pm 0.29 f$ & 74.1 \\
\hline & $1 \times 10^{9}$ & $24.0 \pm 0.19 f-i$ & 76.0 & $20.0 \pm 0.11 \mathrm{~g}$ & 80.0 \\
\hline \multirow[t]{5}{*}{ S. cerevisiae } & $1 \times 10^{5}$ & $54.4 \pm 0.78 b$ & 45.6 & $47.1 \pm 0.80 \mathrm{bc}$ & 52.9 \\
\hline & $1 \times 10^{6}$ & $47.9 \pm 0.44 c$ & 52.1 & $37.7 \pm 0.17 c-f$ & 62.3 \\
\hline & $1 \times 10^{7}$ & $38.1 \pm 0.81 \mathrm{~d}-\mathrm{g}$ & 61.9 & $29.5 \pm 0.47 \mathrm{e}-\mathrm{g}$ & 70.5 \\
\hline & $1 \times 10^{8}$ & $26.1 \pm 0.79 f-h$ & 73.9 & $22.4 \pm 0.18 f-i$ & 77.6 \\
\hline & $1 \times 10^{9}$ & $19.6 \pm 0.43 \mathrm{gh}$ & 80.4 & $15.3 \pm 0.12 \mathrm{~g}-\mathrm{k}$ & 84.7 \\
\hline \multirow[t]{5}{*}{ S. fimicola } & $1 \times 10^{5}$ & $49.0 \pm 0.89 b-f$ & 51.0 & $41.2 \pm 0.61 \mathrm{c}$ & 58.8 \\
\hline & $1 \times 10^{6}$ & $42.4 \pm 0.57 c-h$ & 57.6 & $34.2 \pm 0.72 d$ & 65.8 \\
\hline & $1 \times 10^{7}$ & $38.1 \pm 0.31 \mathrm{~d}-\mathrm{g}$ & 61.9 & $27.1 \pm 0.43 \mathrm{e}-\mathrm{i}$ & 72.9 \\
\hline & $1 \times 10^{8}$ & $27.2 \pm 0.88 f g$ & 72.8 & $23.6 \pm 0.21 \mathrm{f}-\mathrm{h}$ & 76.4 \\
\hline & $1 \times 10^{9}$ & $21.8 \pm 0.67 f-1$ & 78.2 & $17.7 \pm 0.12 \mathrm{~g}-\mathrm{i}$ & 82.3 \\
\hline \multirow[t]{5}{*}{ G. roseum + S. cerevisiae } & $1 \times 10^{5}$ & $53.3 \pm 0.42 b c$ & 46.7 & $44.8 \pm 0.49 b-e$ & 55.2 \\
\hline & $1 \times 10^{6}$ & $44.6 \pm 0.92 c-f$ & 55.4 & $36.5 \pm 0.41 c-g$ & 63.5 \\
\hline & $1 \times 10^{7}$ & $35.9 \pm 0.21 d-k$ & 64.1 & $27.1 \pm 0.80 \mathrm{e}-\mathrm{i}$ & 72.9 \\
\hline & $1 \times 10^{8}$ & $27.2 \pm 0.73 f g$ & 72.8 & $24.8 \pm 0.87 f g$ & 75.2 \\
\hline & $1 \times 10^{9}$ & $21.8 \pm 0.30 f-1$ & 78.2 & $17.7 \pm 0.36 \mathrm{~g}-\mathrm{i}$ & 82.3 \\
\hline \multirow[t]{5}{*}{ G. roseum + S. fimicola } & $1 \times 10^{5}$ & $50.0 \pm 0.61 b-e$ & 50.0 & $43.6 \pm 0.10 b-f$ & 56.4 \\
\hline & $1 \times 10^{6}$ & $40.3 \pm 0.33 d$ & 59.7 & $36.5 \pm 0.82 \mathrm{c}-\mathrm{g}$ & 63.5 \\
\hline & $1 \times 10^{7}$ & $34.8 \pm 0.31 \mathrm{ef}$ & 65.2 & $27.1 \pm 0.98 \mathrm{e}-\mathrm{i}$ & 72.9 \\
\hline & $1 \times 10^{8}$ & $28.3 \pm 0.81 f$ & 71.7 & $22.4 \pm 0.74 f-i$ & 77.6 \\
\hline & $1 \times 10^{9}$ & $20.7 \pm 0.61 \mathrm{~g}$ & 79.3 & $16.5 \pm 0.31 \mathrm{~g}-\mathrm{j}$ & 83.5 \\
\hline \multirow[t]{5}{*}{ S. cerevisiae + S. fimicola } & $1 \times 10^{5}$ & $34.8 \pm 0.43 \mathrm{ef}$ & 65.2 & $28.3 \pm 0.25 e-h$ & 71.7 \\
\hline & $1 \times 10^{6}$ & $25.0 \pm 0.56 f-j$ & 75.0 & $21.2 \pm 0.22 f-j$ & 78.8 \\
\hline & $1 \times 10^{7}$ & $18.5 \pm 0.39 g-i$ & 81.5 & $13.0 \pm 0.14 h-j$ & 87.0 \\
\hline & $1 \times 10^{8}$ & $10.9 \pm 0.38 i$ & 89.1 & $8.3 \pm 0.10 \mathrm{i}-1$ & 91.7 \\
\hline & $1 \times 10^{9}$ & $4.4 \pm 0.10 k$ & 95.6 & $3.6 \pm 0.07 \mathrm{j}-1$ & 96.4 \\
\hline Control & - & $61.3 \pm 1.18 a$ & - & $56.6 \pm 1.09 a$ & - \\
\hline
\end{tabular}

*Values expressed are mean ( \pm standard error) of five replicates

Values given separately for the soil treatments within each column followed by different letters are significantly different at $p<0.05$ 
tested bio-agents $\left(1 \times 10^{5}, 1 \times 10^{6}, 1 \times 10^{7}, 1 \times 10^{8}\right.$, $1 \times 10^{9}$ spores $/ \mathrm{ml}$ ) were added as a soil drench at the rate of $30 \mathrm{ml}$ per each pot, mixed thoroughly, and kept by moisturizing for 7 days. After a week, the wheat seeds were sown in pots following disinfection with SHS.

For the seed + soil treatments, the pathogen inoculum and the spore suspensions of tested bioagents were added to pots as a soil drench, mixed thoroughly, and kept by moisturizing for 7 days. At the end of a week, disinfected seeds were sprayed by spore suspensions of the tested bio-agents and were sown in pots. The experiment was attempted with an average of 5 independent replicates, compared with the control pots inoculated with a spore suspension of the fungal pathogen only. The study was conducted in a plant growth room under a $16 \mathrm{~h}$ photoperiod cycle at $25^{\circ} \mathrm{C}$. The number of dead seedlings was determined after 15 days, and the effect of the bio-agent treatments on damping-off was calculated using the following formula (Topps and Wain 1957).

Table 5 Effect of different concentrations of the seed + soil treatments on damping-off caused by F. graminearum in wheat cultivars

\begin{tabular}{|c|c|c|c|c|c|}
\hline \multirow[t]{3}{*}{ Seed + soil treatments } & \multirow{3}{*}{$\begin{array}{l}\text { Concentrations } \\
\text { (spores/ml) }\end{array}$} & \multicolumn{4}{|l|}{ Wheat Cultivars } \\
\hline & & \multicolumn{2}{|l|}{ Gun 91} & \multicolumn{2}{|l|}{ Sultan 95} \\
\hline & & Incidence of damping-off (\%) & Inhibition rate (\%) & Incidence of damping-off (\%) & Inhibition rate (\%) \\
\hline \multirow[t]{5}{*}{ G. roseum } & $1 \times 10^{5}$ & $35.3 \pm 0.67 d-k^{*}$ & 64.7 & $29.5 \pm 0.73 e-g^{*}$ & 70.5 \\
\hline & $1 \times 10^{6}$ & $28.3 \pm 0.52 f$ & 71.7 & $22.4 \pm 0.56 f-i$ & 77.6 \\
\hline & $1 \times 10^{7}$ & $19.6 \pm 0.43 \mathrm{gh}$ & 79.3 & $16.5 \pm 0.33 g-j$ & 83.5 \\
\hline & $1 \times 10^{8}$ & $13.1 \pm 0.30 h-k$ & 86.9 & $10.6 \pm 0.21 \mathrm{ij}$ & 88.2 \\
\hline & $1 \times 10^{9}$ & $7.7 \pm 0.18 j$ & 92.3 & $7.1 \pm 0.13 i-m$ & 92.9 \\
\hline \multirow[t]{5}{*}{ S. cerevisiae } & $1 \times 10^{5}$ & $32.7 \pm 0.77 \mathrm{e}-\mathrm{h}$ & 67.3 & $30.6 \pm 0.49 \mathrm{ef}$ & 69.4 \\
\hline & $1 \times 10^{6}$ & $26.1 \pm 0.43 f-h$ & 73.9 & $23.6 \pm 0.63 f-h$ & 76.4 \\
\hline & $1 \times 10^{7}$ & $19.6 \pm 0.65 \mathrm{gh}$ & 80.4 & $13.0 \pm 0.41 \mathrm{~h}-\mathrm{j}$ & 87.0 \\
\hline & $1 \times 10^{8}$ & $10.9 \pm 0.32 i$ & 89.1 & $10.6 \pm 0.37 \mathrm{ij}$ & 89.4 \\
\hline & $1 \times 10^{9}$ & $5.5 \pm 0.11 j-m$ & 94.5 & $4.8 \pm 0.15 \mathrm{jk}$ & 95.2 \\
\hline \multirow[t]{5}{*}{ S. fimicola } & $1 \times 10^{5}$ & $30.5 \pm 0.60 e-j$ & 69.5 & $25.9 \pm 0.50 f$ & 74.1 \\
\hline & $1 \times 10^{6}$ & $21.8 \pm 0.23 f-I$ & 78.2 & $17.7 \pm 0.39 g-i$ & 82.3 \\
\hline & $1 \times 10^{7}$ & $14.2 \pm 0.35 h-j$ & 85.8 & $11.8 \pm 0.26 i$ & 88.2 \\
\hline & $1 \times 10^{8}$ & $9.8 \pm 0.21 i-k$ & 90.2 & $8.3 \pm 0.21 i-1$ & 91.7 \\
\hline & $1 \times 10^{9}$ & $6.6 \pm 0.15 \mathrm{jk}$ & 93.4 & $5.9 \pm 0.11 j$ & 94.1 \\
\hline \multirow[t]{5}{*}{ G. roseum + S. cerevisiae } & $1 \times 10^{5}$ & $26.1 \pm 0.34 f-h$ & 73.9 & $24.8 \pm 0.67 f \mathrm{~g}$ & 75.2 \\
\hline & $1 \times 10^{6}$ & $15.3 \pm 0.27 \mathrm{hi}$ & 84.7 & $16.5 \pm 0.32 \mathrm{~g}-\mathrm{j}$ & 83.5 \\
\hline & $1 \times 10^{7}$ & $10.9 \pm 0.21 i$ & 89.1 & $9.5 \pm 0.24 i-k$ & 90.5 \\
\hline & $1 \times 10^{8}$ & $7.7 \pm 0.18 j$ & 92.3 & $5.9 \pm 0.18 j$ & 94.1 \\
\hline & $1 \times 10^{9}$ & $3.3 \pm 0.05 k-m$ & 96.7 & $2.4 \pm 0.12 k$ & 97.6 \\
\hline \multirow[t]{5}{*}{ G. roseum + S. fimicola } & $1 \times 10^{5}$ & $29.4 \pm 0.89 e-j$ & 70.6 & $27.1 \pm 0.31 \mathrm{e}-\mathrm{i}$ & 72.9 \\
\hline & $1 \times 10^{6}$ & $21.8 \pm 0.54 f-1$ & 78.2 & $20.0 \pm 0.22 \mathrm{~g}$ & 80.0 \\
\hline & $1 \times 10^{7}$ & $15.3 \pm 0.41 \mathrm{ij}$ & 84.7 & $14.2 \pm 0.27 \mathrm{hi}$ & 85.8 \\
\hline & $1 \times 10^{8}$ & $10.9 \pm 0.43 i$ & 89.1 & $9.5 \pm 0.15 i-k$ & 90.5 \\
\hline & $1 \times 10^{9}$ & $5.5 \pm 0.21 j-m$ & 94.5 & $4.8 \pm 0.11 \mathrm{jk}$ & 95.2 \\
\hline \multirow[t]{5}{*}{ S. cerevisiae + S. fimicola } & $1 \times 10^{5}$ & $9.8 \pm 0.12 i-k$ & 90.2 & $7.1 \pm 0.09 i-m$ & 92.9 \\
\hline & $1 \times 10^{6}$ & $7.7 \pm 0.09 j$ & 92.3 & $4.8 \pm 0.07 \mathrm{jk}$ & 95.2 \\
\hline & $1 \times 10^{7}$ & $6.6 \pm 0.05 j k$ & 93.4 & $4.8 \pm 0.05 j \mathrm{j}$ & 95.2 \\
\hline & $1 \times 10^{8}$ & $3.3 \pm 0.05 k-m$ & 96.7 & $2.4 \pm 0.02 k$ & 97.6 \\
\hline & $1 \times 10^{9}$ & $1.1 \pm 0.03 m$ & 98.9 & $1.2 \pm 0.02 \mathrm{kl}$ & 98.8 \\
\hline Control & - & $61.3 \pm 1.23 a$ & - & $56.6 \pm 1.12 a$ & - \\
\hline
\end{tabular}




$$
I \%=[(C-T / C)] \times 100
$$

where I \% = inhibition rate, $\mathrm{C}=$ average of nonemerged seedlings in control treatment, and $\mathrm{T}=$ average of non-emerged seedlings after bio-agent treatments.

\section{Statistical analysis}

Statistical analysis of data was subjected to ANOVA (one-way analysis of variance). Significant differences ( $p$ $<0.05)$ were tested by the general linear model (GLM) procedure, using the Duncan's multiple range test (DMRT) for disease incidence, mycelial growth, and lesion length of the pathogenic fungus after bio-agent treatments.

\section{Results and discussion}

The present study is the first report indicating that $G$. roseum, S. cerevisiae, and S. fimicola, and in particular, the mixture of $S$. cerevisiae $+S$. fimicola was promising and effective against the cereal damping-off caused by $F$. graminearum in wheat cultivars.

\section{In vitro antagonistic activity of the bio-agents}

The selected isolates of G. roseum, S. cerevisiae, and S. fimicola showed the highest antagonistic activity inhibiting the growth of $F$. graminearum at a concentration of $1 \times 10^{9}$ spores/ml with an inhibition rate of 84,88 , and $91 \%$, respectively. Accordingly, the decrease of mycelial growth of the pathogen was observed by increasing concentrations, particularly at $1 \times 10^{9}$ spores $/ \mathrm{ml}$, in comparison with the lower concentrations (Table 1, Fig. 1). The antagonistic activity of the bio-agents was similar to the in vitro studies as G. roseum inhibited growth of $F$. graminearum (Dubey et al. 2014), F. culmorum, F. graminearum, and $F$. nivale and caused degradation of hyphal walls (Pisi et al. 2001), S. cerevisiae and S. fimicola inhibited mycelial growth of Fusarium oxysporum (Manoch et al. 2008 and Shalaby and El-Nady 2008).

\section{In vivo antagonistic activity of the bio-agents} Antagonistic activity of $G$. roseum against $F$. graminearum In the present study, G. roseum had a high antagonistic activity against the pathogen growth in both wheat cultivars at a concentration of $1 \times 10^{9}$ spores $/ \mathrm{ml}$ of the foliar,

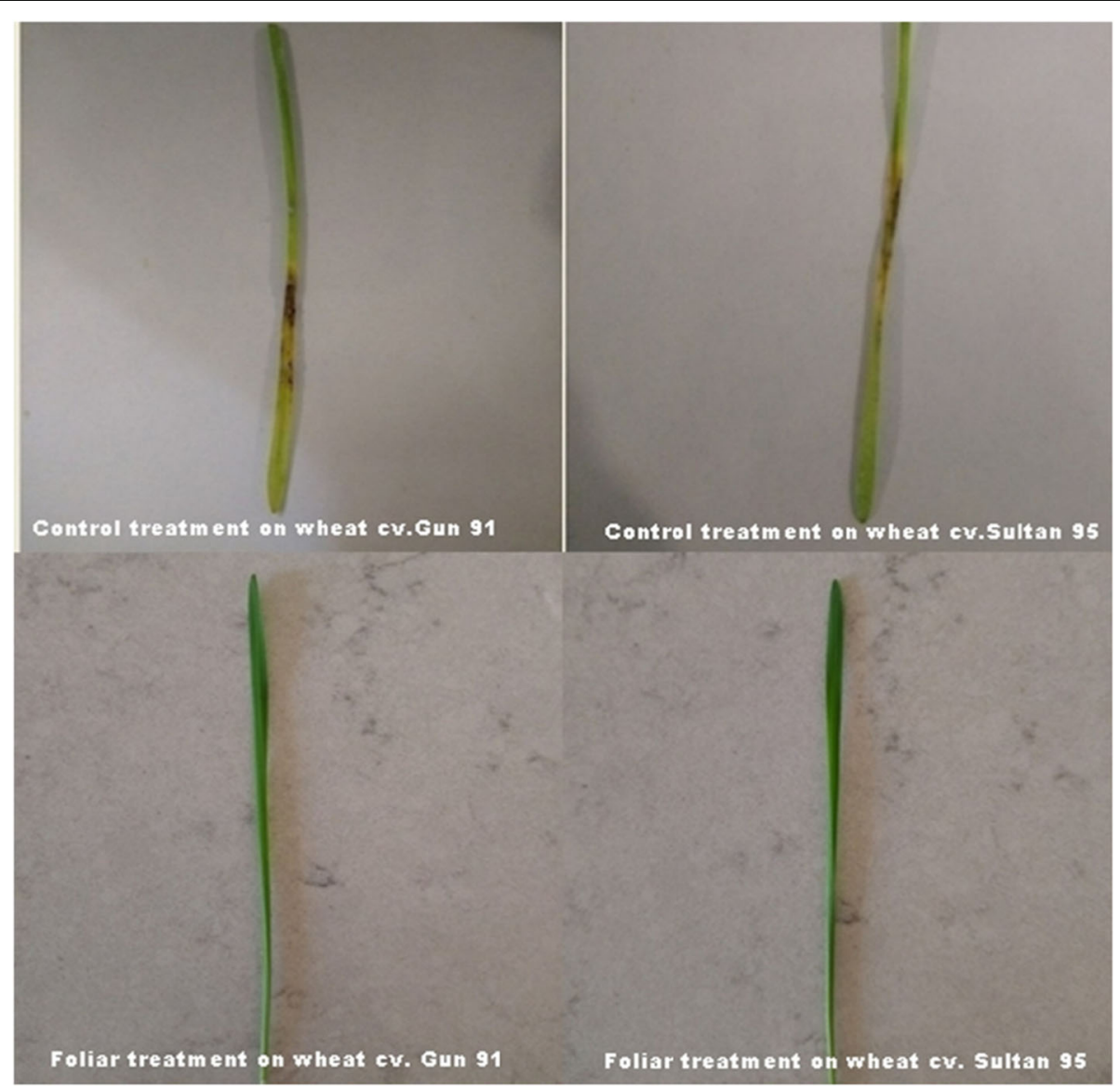

Fig. 2 The leaf infection caused by F. graminearum in wheat cultivars after the foliar treatments with S. cerevisiae + S. fimicola at $1 \times 10^{9}$ spores/ $\mathrm{ml}$ and the control treatments 
soil, and seed treatments with an inhibition rate above $76 \%$ (Tables 2, 3, and 4); and the seed + soil treatment in particular exhibited an inhibition rate above 92\% (Table 5). These are in agreement with data obtained by other researches, which indicated that G. roseum stimulated plant growth and decreased growth of F. culmorum in winter wheat (Knudsen et al. 1995), and Gliocladium spp. reduced the colonization of Fusarium species in wheat and maize plants (Luongo et al. 2005 and Woo et al. 2006). When taking into account that G. roseum had biosynthetic gene clusters to encode a large number of enzyme complexes and secondary metabolites, reported by Karlsson et al. (2015) and Sun et al. (2015), it was suggested that the main reason of antagonistic activity was related to the production of antifungal substances such as enzymes and secondary metabolites. In this manner, as a result of the interaction among them, G. roseum might damage to the cell structure along with hyphal penetration and promote disruption of pathogen cell.

\section{Antagonistic activity of $S$. cerevisiae against $F$. graminearum}

The increment of $S$. cerevisiae concentration resulted in a decrease of the disease incidence, and inhibition rates of the yeast at a concentration of $1 \times 10^{9}$ spores $/ \mathrm{ml}$ of the foliar, soil, and seed treatments were determined to be above $80 \%$ for both wheat cultivars (Tables 2, 3, and 4). However, at the seed + soil treatment, at a $1 \times 10^{9}$ spores $/ \mathrm{ml}$ concentration of the yeast, the inhibition rate reached to approximately $95 \%$ for both wheat cultivars (Table 5). Obtained findings were in agreement with Shalaby and El-Nady (2008), who reported that $F$. oxysporum was adequately controlled by S. cerevisiae at $5 \mathrm{gl}^{-1}\left(2.78 \times 10^{8} \mathrm{cell} / \mathrm{ml}\right)$ and that incidence of damping-off was $6.67 \%$. Based on a study conducted by Attyia and Youssry (2001) related to indole3 -acetic acid (IAA) producing ability of S. cerevisiae against Fusarium spp. in tomato and eggplant seedlings; it was supposed that the yeast treatments could exhibit plant growthpromoting activity by releasing IAA, which improved plant resistance to F. graminearum, and suppressed therefore the pathogen growth. So it was concluded that $S$. cerevisiae increased the division and expansion of plant cells, stimulated plant growth parameters, and consequently decreased the disease incidence.

\section{Antagonistic activity of S. fimicola against $F$. graminearum}

The antagonistic activity of $S$. fimicola at a concentration of $1 \times 10^{9}$ spores $/ \mathrm{ml}$ of the soil treatment showed

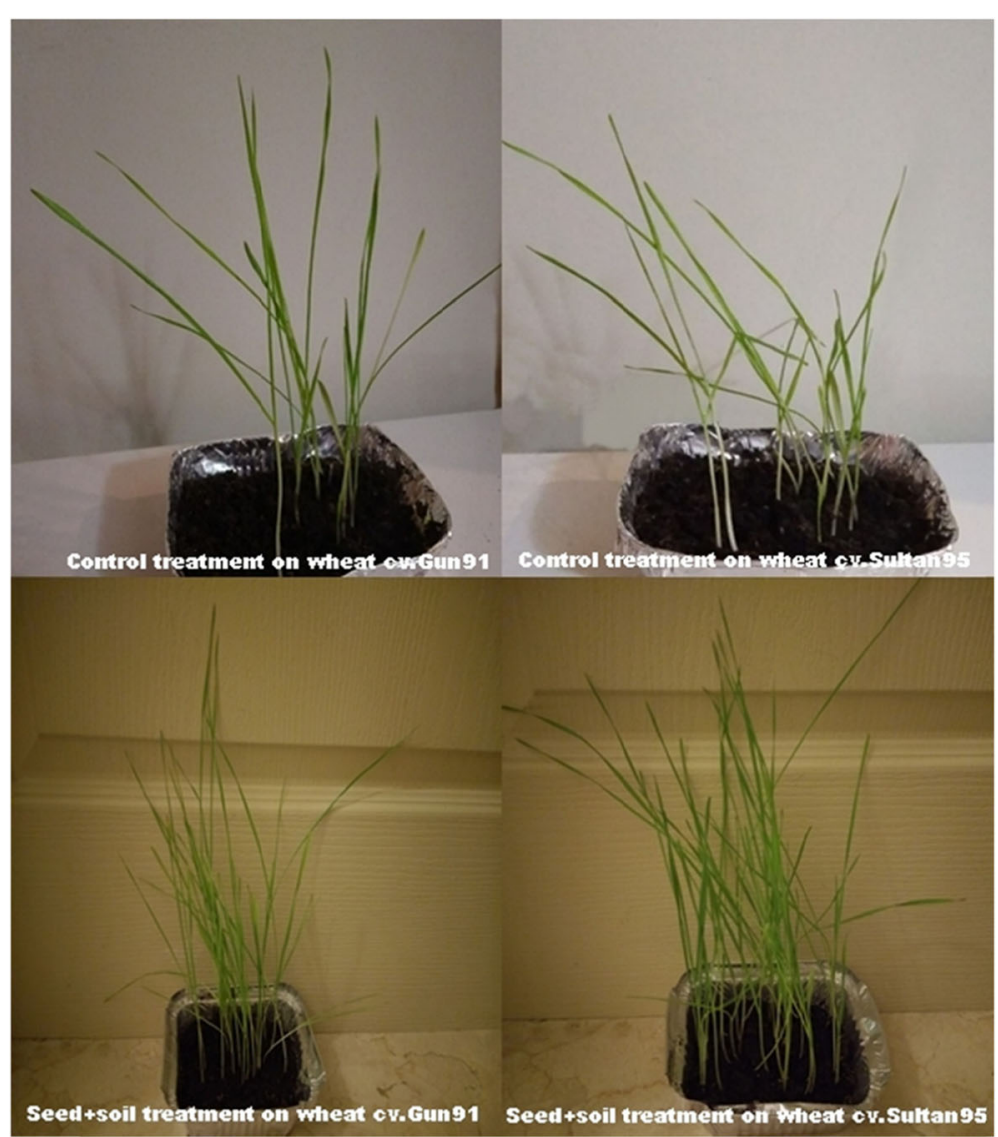

Fig. 3 Damping-off caused by F. graminearum in wheat cultivars after the seed + soil treatments with S. cerevisiae + S. fimicola at $1 \times 10^{9}$ spores/ $\mathrm{ml}$ and the control treatments 
relatively high rates of inhibition (above 78\%), following the foliar and seed treatments with an inhibition rate above $83 \%$ for both wheat cultivars (Tables 2, 3, and 4). Moreover, the seed + soil treatment at the same concentration showed an inhibition rate above 90\% against growth of F. graminearum (Table 5). So far, the antagonistic activity of $S$. fimicola was only reported in maize against F. graminearum under greenhouse conditions by Abdallah et al. (2018), who found that it inhibited growth of F. graminearum and reduced production of zearalenone. Based on a previous study by Dewan et al. (1994), the antagonistic activity was attributed to release compounds (triacontanol and indole-3-carboxaldehyde) from $S$. fimicola, acting as natural growth stimulators to contribute the plant growth and the reduction of disease incidence of Gaeumannomyces graminis var. tritici. In the present study, the antagonistic activity of S. fimicola and its supportive effect on plant development against the pathogenic fungus were probably related to production of these bioactive compounds.

\section{Antagonistic activity of the mixture of the bio-agents against $F$. graminearum}

When evaluating mixture of the bio-agents, the foliar (Fig. 2), seed and soil treatments of $S$. cerevisiae $+S$. fimicola at a concentration of $1 \times 10^{9}$ spores $/ \mathrm{ml}$ exhibited an antagonistic activity with an inhibition rate above $87 \%$ for both wheat cultivars (Tables 2, 3, and 4). In addition, it was determined that the seed + soil treatment of $S$. cerevisiae $+S$. fimicola had an antagonistic activity by over $90 \%$ even at a concentration of $1 \times 10^{5}$ spores/ml; and the pathogen growth was inhibited almost completely at concentrations of $1 \times 10^{8}$ and $1 \times$ $10^{9}$ spores/ml with an inhibition rate above $96 \%$ (Table 5, Fig. 3). It was concluded that there was a synergistic interaction and almost no antagonistic activity between $S$. cerevisiae and S. fimicola. Indeed, in vitro results confirmed that they had approximately similar diameters of the mycelial growth $(5.5 \mathrm{~cm}$ for $S$. cerevisiae and $5.8 \mathrm{~cm}$ for S. fimicola, respectively) on PDA medium. However, the seed + soil treatment of G. roseum + S. fimicola and G. roseum $+S$. cerevisiae showed similar results with all single treatments of G. roseum, S. cerevisiae, and S. fimicola. It was attributed that the released compounds from S. fimicola and S. cerevisiae probably suppressed the growth of G. roseum in the same way as in vitro results. Therefore, there was no synergistic interaction between G. roseum and other bio-agents against F. graminearum, emphasizing that $G$. roseum showed a poor mycelial growth with diameters of 3.5 and $3.0 \mathrm{~cm}$ against $S$. cerevisiae and S. fimicola, respectively. It was considered that a combination of the seed and soil treatments containing S. cerevisiae + S. fimicola at a concentration of $1 \times 10^{9}$ spores/ml had a synergistic effect on antagonistic activity against F. graminearum.

\section{Conclusion}

The isolates of G. roseum, S. cerevisiae, and S. fimicola and, in particular, the mixture of $S$. cerevisiae $+S$. fimicola had a high capacity in terms of antagonistic activity at increasing concentrations against F. graminearum in both wheat cultivars. It is supposed that application of biological preparations containing these bio-agents will be significantly promising and applicable as an alternative tool to synthetic chemicals and other low-efficiency or harmful methods for the control of damping-off caused by F. graminearum.

\section{Abbreviations}

IAA: Indole-3-acetic acid; PDA: Potato dextrose agar; RBA: Rose Bengal Agar; SDW: Sterile distilled water; SHS: Sodium hypochlorite solution

\section{Acknowledgements}

The author is grateful to Julia SAHRAN and Nur SIVRI for providing language support and useful remarks.

\section{Author's contributions}

I am the only author of this manuscript. So I am responsible for all the steps. The author read and approved the final manuscript.

Funding

No funding.

Availability of data and materials

Not applicable.

Ethics approval and consent to participate

Not applicable.

Consent for publication

Not applicable.

\section{Competing interests}

The author declares that he has no competing interests.

Received: 24 September 2019 Accepted: 3 April 2020

Published online: 20 April 2020

\footnotetext{
References

Abdallah MF, De Boevre M, Landschoot S, De Saeger S, Haesaert G, Audenaert K (2018) Fungal endophytes control Fusarium graminearum and reduce trichothecenes and zZearalenone in maize. Toxins (Basel) 10(12):24

Attyia SH, Youssry AA (2001) Application of Saccharomyces cerevisiae as a biocontrol agent against some diseases of Solanaceae caused by Macrophomina phaseolina and Fusarium solani. Egypt J of Biol 3:79-87

Avelino J, Cristancho M, Georgiou S, Imbach P, Aguilar L, Bornemann G (2015) The coffee rust crises in Colombia and Central America. Impacts, plausible causes, and proposed solutions. Food Security 7(2):303-321

Booth C (1971) The genus Fusarium. Com. Myc. Inst. Kew, Surrey, England, p 237

Booth C (1977) Fusarium. Laboratory guide to the identification of the major species. Commonwealth Mycological Inst. Kew, Surrey, England, p 58

Burgess L, Summerell BA, Bullock S, Gott PG and Backhouse D (1994) Laboratory manual for Fusarium research. 3rd Ed. Fusarium Research Lab. The University of Sydney, p 133.

Dewan MM, Ghisalbertib EL, Rowland C, Sivasithamparam K (1994) Reduction of symptoms of take-all of wheat and rye-grass seedlings by the soil-borne fungus Sordaria fimicola. Appl Soil Ecol 1:45-51

Domsch KH, Gams W, Anderson TH (1980) Compendium of soil fungi. Academic Press, London, New York, Toronto, Sydney, San Francisco, 1:859.
} 
Dubey MK, Jensen DF, Karlsson M (2014) An ATP-binding cassette pleiotropic drug transporter protein is required for xenobiotic tolerance and antagonism in the fungal biocontrol agent Clonostachys rosea. Mol Plant Microbe Interact 27:725-732

Ellis MB (1971) Dematiaceus hyphomycetes. Comm. Mycol. Inst, Kew, Surrey, England, p 608

Ellis MB (1976) More dematious hyphomycetes. Commonwealth Mycol Inst. England, p 507

Gams W, Bissett J (1998) In: Kubicek C, Harman G (eds) Morphology and identification of Trichoderma. Gliocladium and Trichoderma. 1. Taylor and Francis, London, pp 3-34

Hue AG, Voldeng HD, Savard ME, Fedak G, Tian X, Hsiang T (2009) Biological control of Fusarium head blight of wheat with Clonostachys rosea strain ACM941. Can J Plant Pathol 31:169-179

Hussein MS (1973) Pathological studies on root rot disease of peas (Pisum sativum). M. Sc. Thesis. Fac Agric Al-Azhar Univ, Egypt, Google Scholar

Jalaluldeen AM, Sijam K, Othman R, Ahmad ZAM (2014) Isolation and characterization of actinomycetes with in-vitro antagonistic activity against Fusarium oxysporum from rhizosphere of chilli. International Journal of Enhanced Research in Science Technology \& Engineering 3(12):54-61

Jogaiah S, Abdelrahman M, Tran LSP, Ito SI (2018) Different mechanisms of Trichoderma virens-mediated resistance in tomato against Fusarium wilt involve the jasmonic and salicylic acid pathways. Plant Pathol 19(4):870-882

Joshi SM, De Britto S, Jogaiah S, Ito SI (2019) Mycogenic selenium nanoparticles as potential new generation broad spectrum antifungal molecules. Biomolecules 9(9):419

Karaca I (1974) Sistematik Bitki Hastalıkları, Deuteromycetes (Fungi Imperfecti) Cilt IV. Ege Üniv. Zir. Fak. Yayınları, No: 217.

Karlsson M, Durling MB, Choi J, Kosawang C, Lackner G, Tzelepis GD (2015) Insights on the evolution of mycoparasitism from the genome of Clonostachys rosea. Genome Biol Evol 7:465-480

Kawasaki Y (2008) Aminoglycosides and syringomycin E as fungicides against Fusarium graminearum in head blight disease. Master Thesis. Utah State University, p77

Kelly AC, Ward TJ (2018) Population genomics of Fusarium graminearum reveals signatures of divergent evolution within a major cereal pathogen. PLoS ONE 13:e0194616

Knudsen IMB, Hockenhull J, Jensen DF (1995) Biocontrol of seedling diseases of barley and wheat caused by Fusarium culmorum and Bipolaris sorokiniana: effects of selected fungal antagonists on growth and yield components. Plant Pathol 44:467-477

Landschoot S, Audenaert K, Waegeman W, de Baets B, Haesaert G (2013) Influence of maize-wheat rotation systems on Fusarium head blight infection and deoxynivalenol content in wheat under low versus high disease pressure. Crop Prot 52:14-21

Leplat J, Friberg H, Abid M, Steinberg C (2013) Survival of Fusarium graminearum, the causal agent of Fusarium head blight. A review. Agron Sustain Dev 33:97-111

Lundquist N (1972) Nordic Sordariaceae. Symbolae botanicae upsalienses 20(1):1-374

Luongo L, Galli M, Corazza L, Meekes E, De Haas L, Van Der Plas CL, Köhl J (2005) Potential of fungal antagonists for biocontrol of Fusarium sp. in wheat and maize through competition in crop debris. Biocontrol Sci Tech 15:229-242

Manoch L, Jeamjitt O, Dethoup T, Kokaew J, Eamvijarn A, Visarathanonth N, Chamswarng C (2008) Biological control of plant pathogenic fungi using Talaromyces flavus, Sordaria fimicola and some endophytic fungi. Phytopathology 98(6):97

Nelson PE, Toussoun TA, Cook RJ (1981) Fusarium: diseases biology, and taxonomy. University Park, PA Pennsylvania State University Press, pp 64-76

Omotayo OP, Omotayo AO, Mwanza M, Babalola OO (2019) Prevalence of mycotoxins and their consequences on human health. Toxicol Res 35:1-7

Peltonen-Sainio P, Jauhiainen L, Laurila IP (2009) Cereal yield trends in northern European conditions: changes in yield potential and its realization. Field Crops Research 110:85-90

Perez C, Dill-Macky R, Kinkel LL (2008) Management of soil microbial communities to enhance populations of Fusarium graminearum-antagonists in soil. Plant Soil 302:53-69

Pisi A, Roberti R, Zakrisson E, Filipini G, Mantovani W, Cesari A (2001) SEM investigation about hyphal relationships between some antagonistic fungi against Fusarium spp. footrot pathogen of wheat. Phytopathol Mediterr 40(1): $37-44$
Rai MK, Kovics GJ (2010) Progress in mycology. The Netherlands, pp75-94 Rasiukeviciute N, Kelpsiene J (2018) The impact of Fusarium graminearum infection on different plant seeds. Research for Rural Development 2:114-118

Satapute P, Kamble MV, Adhikari SS, Jogaiah S (2019) Influence of triazole pesticides on tillage soil microbial populations and metabolic changes. Sci Total Environ 651:2334-2344

Schaafsma AW, Tamburic llincic L, Hooker DC (2005) Effect of previous crop, tillage, field size, adjacent crop, and sampling direction on airborne propagules of Gibberella zeae/Fusarium graminearum, fusarium head blight severity, and deoxynivalenol accumulation in winter wheat. Canadian journal of plant pathology 27(2):217-224

Shalaby ME, El-Nady MF (2008) Application of Saccharomyces cerevisiae as a biocontrol agent against Fusarium infection of sugar beet plants. Acta Biologica Szegediensi 52(2):271-275

Singh K, Frisvard JC, Thrane U, Mathur SB (1991) An illustrated manual on identification of some seed-borne aspergilli, fusaria, penicillia, and their mycotoxins. Danish Gow Inst of Seed Path For Developing Countries Denmark, p 233

Sun ZB, Sun MH, Li SD (2015) Identification of mycoparasitism-related genes in Clonostachys rosea 67-1 active against Sclerotinia sclerotiorum. Sci Rep 5:18169

Suzzi G, Komano P, Ponti I, Montuschi C (1995) Natural wine yeasts as biocontrol agents. Journal of Applied Bacteriology 78(3):304-308

Topps JH, Wain RL (1957) Investigation on fungicides. III. The fungi toxicity of 3and 5- alkyl salicylanilide and P-chloronilines. Ann Appl Biol 45(3):506-511

Toussoun TA, Nelson PE (1995) A pictorial guide to the identification of Fusarium species. Fusarium. The Pennsylvania State University Press. University Park and London, p 43

Vaughan Martini A, Martini A (1993) A taxonomic key for the genus Saccharomyces. Syst Appl Microbiol 16:113-119

Vinale F, Ghisalberti EL, Sivasithamparam K, Marra R, Ritieni A (2009) Factors affecting the production of Trichoderma harzianum secondary metabolites during the interaction with different plant pathogens. Lett Appl Microbiol 48 705-711

Woo SL, Scala F, Ruocco M, Lorito M (2006) The molecular biology of interactions between Trichoderma sp., phytopathogenic fungi, and plants. Phytopathology 96:181-185

Zhang L, Yang J, Niu Q, Zhao X, Ye F, Liang L (2008) Investigation on the infection mechanism of the fungus Clonostachys rosea against nematodes using the green fluorescent protein. Appl Microbiol Biotechnol 78:983-990

\section{Publisher's Note}

Springer Nature remains neutral with regard to jurisdictional claims in published maps and institutional affiliations.

\section{Submit your manuscript to a SpringerOpen ${ }^{\circ}$ journal and benefit from:}

- Convenient online submission

- Rigorous peer review

- Open access: articles freely available online

- High visibility within the field

- Retaining the copyright to your article

Submit your next manuscript at $>$ springeropen.com 